\title{
Seminare / Séminaires / Seminari 2008
}

\section{Praxiseröffnung/-übernahme}

\section{Themen}

Juristische Aspekte (Praxisbewilligung, Zulassung zur Sozialversicherung, Vertragswesen), Ehe- und Erbrecht (Trennung Privat- vom Geschäftsvermögen, Ehegüterstand, Erbschaftsfolge/-planung), Praxiseinrichtung (Raumplanung, Inneneinrichtung, Kostenberechnung), Praxisadministration (Leistungserfassungs- und Abrechnungssysteme, Praxismarketing), Unternehmensbewertung einer Arztpraxis (Berechnung Inventarwert und Goodwill als Verhandlungsbasis), Finanzierung einer Arztpraxis (Businessplan, Kreditarten, Absicherungsmöglichkeiten), Versicherungen (Personen- und Sachversicherungen, Vorsorgeplanung).

\section{Sponsoren}

Versicherung der Schweizer Ärzte, Schweizerische Ärzte-Krankenkasse, Druckerei Schmid-Mogelsberg, Labornetz daCapo, Unilabs SA, Labor medica, Labor team w ag, Kern Concept AG und EMH Schweizerischer Ärzteverlag AG.

\section{Daten}

\begin{tabular}{llll} 
K04 & $\begin{array}{l}\text { Donnerstag, 4. September 2008 } \\
\text { Zürich }\end{array}$ & FMT \\
\hline K05 & $\begin{array}{l}\text { Donnerstag, 6. November 2008 } \\
\text { Basel }\end{array}$ & $\begin{array}{l}\text { Hotel } \\
\text { 9.00-16.30 Uhr }\end{array}$ & Victoria \\
\hline
\end{tabular}

\section{Praxisübergabe}

\section{Hinweis}

Vor allem aus steuerplanerischer Sicht lohnt es sich, sich bereits frühzeitig (5-10 Jahre) mit diesem Thema auseinanderzusetzen.

\section{Themen}

Juristische Aspekte (Praxisübergabevertrag, allg. Vertragswesen, Übergabe der Krankengeschichten), Unternehmensbewertung einer Arztpraxis (Berechnung Inventarwert und Goodwill als Verhandlungsbasis), Versicherungen (Übergabe/Auflösung von Versicherungsverträgen, Pensions- und Finanzplanung), Steuern (worauf ist zu achten bei der Übergabe der Praxis, Optimierung der steuerlichen Auswirkungen, Liquidations- und Grundstückgewinnsteuer, Bestimmung des optimalen Übergabezeitpunktes).

\begin{abstract}
Sponsoren
Versicherung der Schweizer Ärzte, Schweizerische Ärzte-Krankenkasse, Labor medica, Labor team w ag, Kern Concept AG und EMH Schweizerischer Ärzteverlag AG.
\end{abstract}

\section{Daten}

K09 Donnerstag, 11. September 2008 FMT Zürich 13.30-18.00 Uhr

K10 Donnerstag, 13. November 2008 Hotel Basel 13.30-18.00 Uhr Victoria

\section{Finanz- und Steuerplanung}

\section{Themen}

Finanzplanung (Businessplan, buchhalterische Massnahmen vor Praxiseröffnung/-übernahme, Standardkontenplan für Ärzte, System der doppelten Buchhaltung, EDV-unterstützte Buchführungslösung), Steuerplanung (Steueraspekte bei Eintritt in die Selbständigkeit, Steuerfallen und Steuerrisiken, optimierte Steuerplanung).

\section{Sponsoren}

Kern Concept AG und EMH Schweizerischer Ärzteverlag AG.

\section{Daten}

$\begin{array}{llll}\text { K13 } & \begin{array}{l}\text { Donnerstag, 18. September 2008 } \\ \text { Zürich }\end{array} & \text { FMT } \\ \text { 13.30-18.00 Uhr } & \\ \text { K14 } & \begin{array}{l}\text { Donnerstag, 20. November 2008 } \\ \text { Basel }\end{array} & \begin{array}{l}\text { Hotel } \\ \text { 13.30-18.00 Uhr }\end{array} & \text { Victoria }\end{array}$

\section{Jahre vor Pensionierung}

\section{Teilnehmende}

Das Seminar richtet sich an Ärztinnen und Ärzte, welche die Pensionierung (auch eine frühzeitige) nicht dem Zufall überlassen wollen.

\section{Inhalt}

Reich in Rente? Behandlung von Fragen zu einem möglichen Kapitalbedarf, zu möglichen Vorsorgelösungen sowie zum steuerlich optimierten Vorgehen. Treuhand und Altersvorsorge? Aufzeigen von Möglichkeiten, wie ein Praxisverkauf sowie eine ideale Altersplanung aufeinander abgestimmt werden können und wie dies in vielen Fällen zu massiven Steuereinsparungen führen kann. Planung der Altersvor- 
sorge! Für die Planung der Altersvorsorge kann der zukünftige Bedarf aufgrund der Planungsmöglichkeiten genau anvisiert werden, und die zukünftigen Einkünfte können in Bezug auf Höhe, Steuern und Sicherheit optimal geplant werden.

\section{Kosten}

Fr. 90.- (inkl. sämtlicher Kursunterlagen und Verpflegung).

\section{Daten}

$\begin{array}{lll}\text { K31 Donnerstag, 28. August } 2008 & \text { Radisson } \\ \text { St. Gallen } 18.00-20.30 \mathrm{Uhr} & \text { SAS Hotel }\end{array}$

\section{Ouverture et reprise d'un cabinet médical}

\section{Contenu}

Business plan (préparation du plan de financement et crédit d'exploitation, financement par la banque), Aménagement (implantation, projet et concept d'aménagement, choix du mobilier, budget), Estimation d'un cabinet (inventaire et goodwill), Administration d'un cabinet médical (dans le cabinet, par la banque), Assurances (toutes les assurances à l'intérieur et autour du cabinet), Passage du statut de salarié à celui d'indépendant et fiscalité.

\section{Sponsors}

Assurance des Médecins Suisses, Caisse-Maladie des Médecins Suisses, Unilabs SA, Viollier SA, Labor team w sa, MCL Medizinische Laboratorien SA et EMH Editions médicales suisses SA.

\section{Dates}

K22

Jeudi 4 septembre 2008 Genève $\quad 17.00-21.00 \mathrm{~h}$

K23

$$
\text { Jeudi } 20 \text { novembre } 2008
$$$$
\text { Sion } \quad 17.00-21.00 \mathrm{~h}
$$

Europa

\section{Remise d'un cabinet médical}

\section{Contenu}

Aspects juridiques (autour du contrat de remise/reprise), Estimation d'inventaire et goodwill d'un cabinet, Assurances (prévoyance, assurances à l'intérieur et autour du cabinet), Conséquences fiscales d'une remise.

\section{Sponsors}

Assurance des Médecins Suisses, Caisse-Maladie des Médecins Suisses, Labor team w sa et EMH Editions médicales suisses SA.
Dates

K25

Jeudi 13 novembre 2008 Hôtel Royal Genève 17.00-20.45 h

\section{Contabilità e fiscalità (Ticino)}

\section{Contenuto}

L'obiettivo di questo seminario è di dare al medico le conoscenze necessarie per utilizzare autonomamente lo strumento contabile e per operare con più efficienza ed economicità, nonché far comprendere al medico il significato della pianificazione fiscale.

Mediante esempi pratici elaborati in gruppo, sarà illustrato in maniera semplice, ma completa il sistema di gestione della contabilità di uno studio medico con effetto anche sulla pianificazione fiscale.

\section{Date}

$\begin{array}{lll}\text { K28 } & \begin{array}{l}\text { Giovedì } 16 \text { ottobre } 2008 \\ \text { Chiasso dalle } 9.00 \text { alle } 16.00\end{array} & \begin{array}{l}\text { FMH } \\ \text { Fiduciaria } \\ \text { Services }\end{array}\end{array}$

\section{Anmeldung und Auskunft / Inscription et information}

Internet unter www.fmhservices.ch oder FMH Consulting Services, Sandra Stadelmann, Burghöhe 1, 6208 Oberkirch, Tel. 04192500 77, Fax 0419210586

\section{Hinweis/Remarque}

Bei sämtlichen Seminaren, bei denen die Kosten teilweise oder gänzlich von Seminarsponsoren gedeckt werden, werden die Teilnehmeradressen den jeweiligen Sponsoren zur Verfügung gestellt. Les adresses des participants aux séminaires dont les coûts sont couverts en partie ou totalement par des sponsors sont communiquées aux sponsors concernés.

\section{Annullierungsbedingungen / \\ Conditions d'annulation}

Bei Abmeldungen oder Fernbleiben werden folgende Unkostenbeiträge erhoben:

Un montant est perçu pour une absence ou une annulation. Il est de:

- Fr. 50.- pro Person ab 14 Tage vor Seminarbeginn / par personne dans les 15 jours avant le début du séminaire;

- Fr. 80.- pro Person ab 7 Tage vor Seminarbeginn oder Fernbleiben / par personne dans les 7 jours avant le début du séminaire. 